\title{
A Perceptual Grouping Approach for Visual Interpolation between Good Continuation and Minimal Path using Tensor Voting
}

\author{
Amin Massad \\ University of Hamburg, Dept. of Computer Science \\ Vogt-Kölln-Str. 30, D-22527 Hamburg, Germany \\ massad@informatik . uni-hamburg. de
}

\begin{abstract}
The completion of fragmented contours is important for several processes of visual perception. However, the shape of the interpolated curves is not unique but rather varies with the completion type (amodal/modal) and contextual information. The solutions represent a balancing between the two constraints of good continuation and minimal path which is controlled by a parameter called GC-MP contrast. In this paper, we propose an integration of such a parameter into the tensor voting approach which has been proposed as a general-purpose model for perceptual grouping mechanisms. The results of this extension are demonstrated on typical configurations of rectilinear and curvilinear fragments.
\end{abstract}

\section{Introduction}

The integration of fragmented contours into larger units plays an important role in different domains of visual perception such as illusory contours [8], contours of occluded shapes [2], spontaneously splitting figures and transparency [9], blind spot completion and cortical or artificial scotomas [18, 20]. Findings from psychophysical, neurophysiological and computational approaches support the identity hypothesis by [9, 22] that these different domains rely on a common general-purpose process for visual interpolation. In accordance with this concept, three perceptual integration types, for which most studies have emphasised the differences, are unified by [3] into three cases with common geometric aspects but decreasing degrees of phenomenal presence with regards to shape unity: modal completion, amodal completion, and virtual lines. Modal completion, which occurs for illusory contours, creates a strong sensory experience of completeness so that modally completed contours are perceived phenomenologically identical to physically completed objects. In contrast, amodal completion, which occurs for occluded contours, creates the unity between occluded parts and a boundary for the partially occluded region but does not have a real phenomenological presence. The weakest unity is perceived for virtual lines which implicitly connect isolated dots [8] or oriented elements [4, 10].

Still, the identity hypothesis remains disputed by studies which focus on differences between the processes, mainly between modal and amodal completion: It has been as- 
sumed that modal completion is located at early visual processing stages while the phenomenally non-visual amodal completion has been located at higher cortical areas. However, recent findings reviewed in [1] show that V1 and V2 neurons not only respond to real and modal contours as shown by $[11,15,19]$, but also to amodal features. On the other hand, the experiments by [15] revealed that responses to modally completed contours increase along the visual pathway and are strongest in higher visual areas, V3A, V7, $\mathrm{V} 4 \mathrm{v}$, and V8. A unifying explanation of the seemingly contradicting findings is provided by [12] who proposes that both early and late processes are involved in modal completion. According to this model, rapid feed-forward computation propagates from early to later stages, i.e. up to the inferotemporal cortex (IT), where rough hypotheses about the shapes in the visual input are made and fed back down the visual pathway. Hence, the retinotopical layout and high resolution of V1 and V2 are utilised to reconstruct the completed contours more precisely which in turn yields to the perception of the illusory figure in higher visual areas.

Apart from the location of both completion types, a recent study by [23] reveals that the same inducers create different shapes if they are completed modally or amodally: In case of amodal completion, contours were perceived more angular (i.e. with sharper angles) than in case of modal completion. Even for the same type of completion, it has been shown that the shape of the interpolation changes in dependency of the context of the inducers such as retinal gap, orientation, contour polarity and global shape [3].

However, these findings do not lead to the rejection of the identity hypothesis but rather to a reformulation as a weak identity hypothesis: The two completion types are still assumed to be based on the same interpolation mechanism which involves a free parameter to change the smoothing level of the interpolation.

In this paper, we focus on the integration of such a free parameter into the tensor voting framework proposed by Medioni et al. $[6,14]$ as a general-purpose model for perceptual grouping mechanisms. This extension is motivated by the contour interpolation model by Fantoni \& Gerbino [3] which introduces a parameter called GC-MP contrast to balance between the Gestalt principles of good continuation (GC) and minimal path (MP). In contrast to [3], our model does not require geometrical constructions rather it is based on local operations which are biologically more plausible and moreover allow the combination of more than two inducer elements for grouping. Furthermore, the use of tensor fields instead of vector fields allows the simultaneous encoding of orientation and orientation uncertainty.

\section{Related Work}

Our approach belongs to the class of strong models for visual interpolation which do not only indicate whether two fragments may be grouped together but even yield a solution for the interpolated trajectory. Although there exist different approaches belonging to this class, such as elastica, splines, pairs of circular arcs [25], a circular arc and straight line [9], stochastic completion field models [26], relaxation labelling [17], and perceptual saliency theories [21,7], none of these models allows the variation of the interpolated shape. However, as discussed in Sect. 1, the interpolated curve should vary in dependency of contextual information or the completion type. The model of [3] is intended to introduce such a variation. It utilises the combination of two vector-fields, one for the 

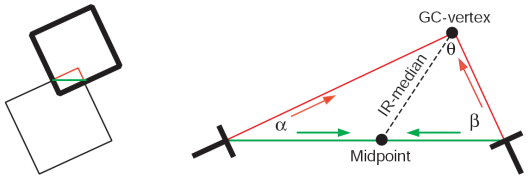

(a)

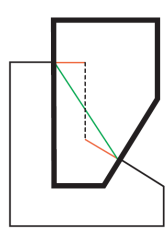

(b)

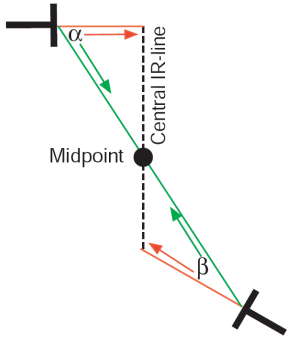

Figure 1: Generic cases of inducer configurations, here for rectilinear fragments (from [3]): (a) Opposite GC-MP angles with intersecting GC-lines. All possible interpolations of the occluded shape (thin outline) lie inside the triangle between the GC and the MP solution. (b) Alternate GC-MP angles with non-intersecting GC-lines. Interpolations lie inside the two triangles forming a so-called bow tie. The central IR-line is constructed as the normal to the GC-line of the larger angle going through the midpoint of the MP-line.

good continuation (GC) solution and one for the minimal path (MP) solution, where the relation between the weights of the two fields is controlled by a parameter called GCMP contrast $\Delta_{G C-M P}:=\frac{w_{G C}-w_{M P}}{w_{G C}+w_{M P}}$ with values in the range from -1 to 1 . The approach requires the construction of the interpolation triangles depicted in Fig. 1.

We employ the tensor voting technique, which has originally been introduced by [6], because it has the several advantages over similar methods: The method is not iterative (although iterations may be utilised [5]), does not require manual setting of starting parameters, handles the presence of multiple curves, regions, and surfaces at the same time, it is highly stable against noise and still preserves discontinuities. In [14], the universality of tensor voting has been demonstrated by application to various early vision problems. The integration of GC-MP contrast into the tensor voting framework is especially important for its application to long-range connections in the context of modal and amodal completion $[13,16]$.

\section{Integration of GC-MP contrast into Tensor Voting}

\subsection{Review of the Tensor Voting Framework}

In this section, we briefly review the tensor voting framework which has been introduced by Medioni et al. [6, 14] as a perceptual saliency model for grouping and segmentation. It is based on the representation of input and output tokens by symmetric tensors which encode local image features simultaneously as orientation and orientation uncertainty. In the $2 \mathrm{D}$-case, a tensor over $\mathbb{R}^{2}$ can be denoted by a symmetric $2 \times 2$ matrix $T=\lambda_{1} \vec{e}_{1} \vec{e}_{1}^{\top}+\lambda_{2} \vec{e}_{2} \vec{e}_{2}^{\top}$ where $\vec{e}_{1}, \vec{e}_{2}$ are the two perpendicular normalised eigenvectors with the corresponding real eigenvalues $\lambda_{1}>\lambda_{2}$. The decomposition of a tensor $T=\left(\lambda_{1}-\lambda_{2}\right) \vec{e}_{1} \vec{e}_{1}^{\top}+\lambda_{2}\left(\vec{e}_{1} \vec{e}_{1}^{\top}+\vec{e}_{2} \vec{e}_{2}^{\top}\right)$ yields to the definition of two saliency measures: The weighting factor $\left(\lambda_{1}-\lambda_{2}\right)$ is called curve- or stick-saliency because it represents an orientation in the direction of the eigenvector $\vec{e}_{1}$. The second weight $\lambda_{2}$ is called junctionor ball-saliency because it is applied to a circle and measures the coincidence of multiple orientations, i.e. the confidence in the presence of a junction. 


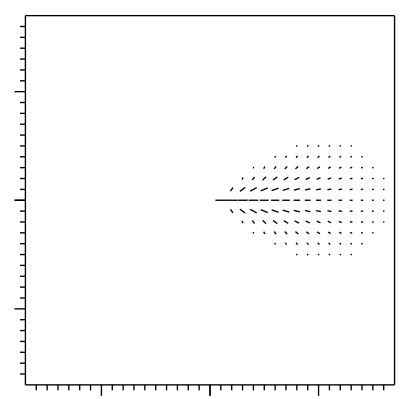

(a)

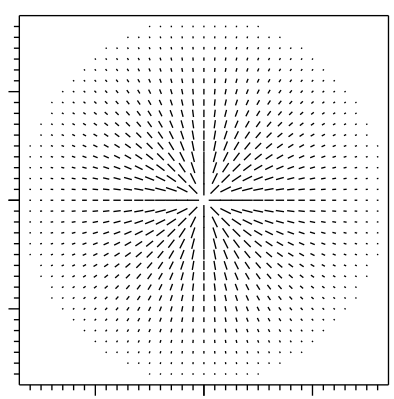

(b)

Figure 2: The basic voting fields (for $\sigma=8$ ): (a) Stick field, semilobe (cf. [13]) for a horizontal unit stick $T_{S}=[1,0][1,0]^{\top}$ located at the origin $O$ and with a first order component pointing rightwards $\vec{v}=[1,0]$ (b) Ball field for a unit ball token $T_{b}=[1,1][1,1]^{\top}$ at $O$.

Grouping of tokens is achieved by the use of voting fields which are applied to the input tokens in a convolution-like manner and combined by tensor addition. For oriented tokens the stick field (Fig. 2a) is applied to each input token by alignment to its eigenvector $\vec{e}_{1}$ and weighting with $\lambda_{1}-\lambda_{2}$. The layout of the stick field is designed to create groupings with neighbouring tokens by strengthening locally collinear or co-circular structures. The votes cast by a horizontal unit stick $T_{S}$ at the origin $O$ to a point $P$ are defined by the stick field as:

$$
\mathbf{S}(P):=D F(r, \theta, \sigma)[-\sin (2 \theta), \cos (2 \theta)]^{\top}[-\sin (2 \theta), \cos (2 \theta)]
$$

where $\theta$ is the angle between the $T_{s}$ and $O P, r=\|O P\|$, and $D F$ a decay function with the scale parameter $\sigma$. In [14], the decay function is defined as $D F(r, \theta, \sigma)=$ $\exp \left(-\frac{s(r, \theta)^{2}+c \kappa(r, \theta)^{2}}{\sigma^{2}}\right)$ where $s(r, \theta)=\theta r / \sin (\theta)$ is the arc length of the circle through $P$ and tangent to $T_{s}, \kappa=2 \sin (\theta) / r$ is its curvature, and $c$ is a scale-dependent constant. As proposed by [7], we use the decay function $D F(r, \theta, \sigma)=\exp \left(-\frac{1}{2} \frac{r^{2}}{\sigma^{2}}\right) \cdot \cos ^{2 k}(\theta)$ with $k=4$ which decouples the functions for $r$ and $\theta$ and does not require the constant $c$. For $\theta>45^{\circ}$, the decay function is set to $D F(r, \theta, \sigma)=0$ because the assumed circular connection does not fulfil the minimal total curvature constraint (an elliptic connection would yield lower total curvature). Note, that in both formulations there is only one free parameter: the scale $\sigma$ which determines the size of the voting field.

For unoriented tokens, where an orientation is (initially) unknown, the ball field (Fig. 2b) is applied which consists of radially aligned tensors whose strength decays with distance from the centre. This inference of orientation is usually applied as a preliminary step on sparse point data before stick-voting can be employed. The votes cast by a unit ball at the origin to a point $P$ is deduced from a full rotation and integration (by tensor addition) of the stick field about the origin. Note that it is equivalent to rotate the field against the receiving point $P$ or to rotate the point $P$ about the origin against the horizontal stick field. Hence, the votes of the ball field at the origin cast to a point $P$ may be defined as $\mathbf{B}(P):=\int_{0}^{2 \pi} \mathbf{S}\left(R_{\theta} P\right) \mathrm{d} \theta$ where $R_{\theta}$ is the rotation matrix for an angle $\theta$ about the origin. In practice, this integration is approximated by a summation over $K$ steps at intervals $2 \pi / K$ :

$$
\mathbf{B}(P) \approx \sum_{i \in[0, K)} \mathbf{S}\left(R_{\theta} P\right) \quad \text { with } \quad \theta=i \frac{2 \pi}{K} .
$$




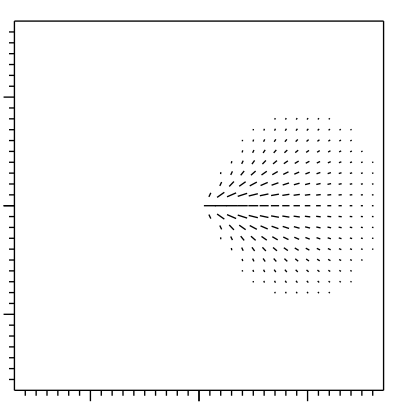

(a)

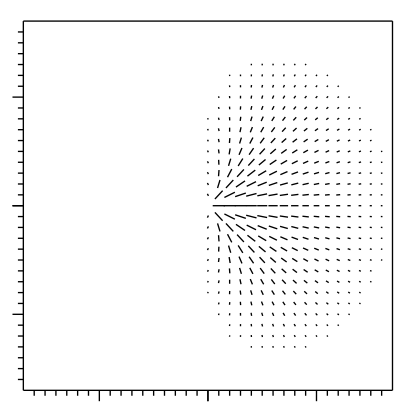

(b)

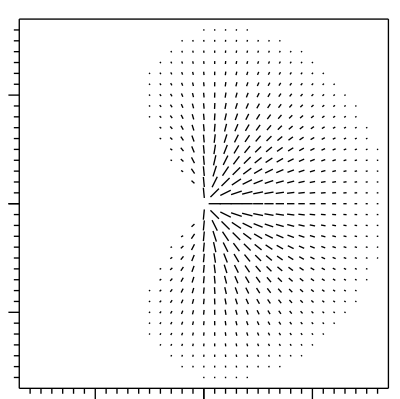

(c)

Figure 3: Newly derived voting fields for varying GC-MP contrasts (for $\sigma=8$ ): (a) $\Delta_{G C-M P}=0.6$ (b) $\Delta_{G C-M P}=0.2$ (c) $\Delta_{G C-M P}=-0.2$. Note that the field for $\Delta_{G C-M P}=1.0$ is depicted in Fig. $2 \mathrm{a}$ and for $\Delta_{G C-M P}=-1.0$ in Fig. 2 b.

For the final result of the grouping process, the maxima are extracted from the saliency maps. The extraction of curves involves a marching algorithm to trace the edgels along their highest saliencies.

\subsection{Integration of GC-MP contrast}

So far, the grouping algorithm depends only on one free parameter: the scale $\sigma$. However, this parameter does not change the layout of the voting fields because the directions of the field tokens remain unchanged while merely the weights of the tokens are changed leading to a larger area of influence. Hence, the parameter $\sigma$ does not change the shape of the interpolated curve (provided that $\sigma$ is large enough to bridge the desired gap size).

In this section, we introduce a solution for the mediation between good continuation and minimal path which is based on the reinterpretation of the basic voting fields presented in Sect. 3.1: The stick field can be interpreted as the representation of a continuation purely based on good continuation $\left(\Delta_{G C-M P}=1.0\right)$, while the ball field represents a continuation purely based on minimal path $\left(\Delta_{G C-M P}=-1.0\right)$. As shown in [14], the stick field is designed to achieve good continuation for an oriented input token (stick tensor) by the four perceptual constraints co-curvilinearity, constancy of curvature, minimal total curvature and proximity. In contrast, the design of the ball field yields straight connections of an input token with neighbouring locations. This behaviour, together with weights decaying with distance, implements a preference for the minimal path between the input token and a point in the neighbourhood. Note that the construction of the minimal path is independent of the orientation of the input token, accordingly the field is isotropic and identical for all input orientations. ${ }^{1}$

In order to achieve intermediate solutions, we utilise the relation (2) between the stick field and the ball fields by replacing the full rotation over $0 \ldots 2 \pi$ with a rotation in the range $-\phi\left(\Delta_{G C-M P}\right) \ldots \phi\left(\Delta_{G C-M P}\right)$ where $\phi\left(\Delta_{G C-M P}\right):=\frac{1-\Delta_{G C-M P}}{2} \pi$ is a function of the GC-MP contrast $\Delta_{G C-M P}$. Then, the definition of a general stick field in dependency of

\footnotetext{
${ }^{1}$ In the original tensor voting framework, the ball field has not been applied to oriented tokens. It has only been used for unoriented tokens, i.e. in case of unknown orientations or the presence of a junction.
} 


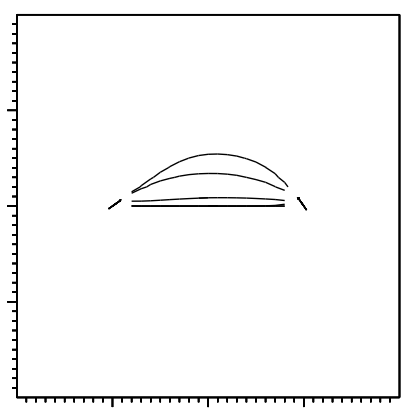

(a)

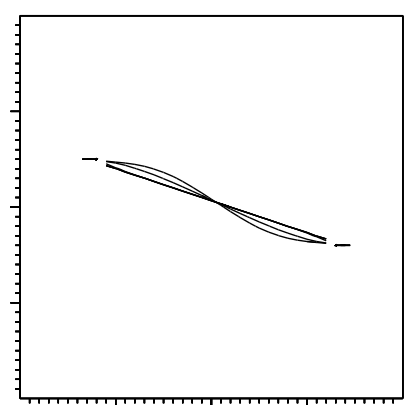

(b)

Figure 4: Interpolated curves for varying GC-MP contrasts $\Delta_{G C-M P}=$ $-1.0,-0.6,-0.2, \ldots, 1.0$ : (a) interpolations between two opposite inducers (left angle $\alpha=35^{\circ}$, right angle $\beta=55^{\circ}$, distance $d=20, \sigma=16$ ), (b) two alternate inducers $\left(\alpha=20^{\circ}, \beta=20^{\circ}, d=30\right)$.

the GC-MP contrast becomes

$$
\mathbf{S}_{G C-M P}(P):=\sum_{i \in[-a, 0] \cup[0, a)} \mathbf{S}\left(R_{\theta} P\right) \quad \text { with } \quad \theta=i \frac{2 \pi}{K}, a=\left\lfloor\phi\left(\Delta_{G C-M P}\right) \frac{K}{2 \pi}\right\rfloor .
$$

According to this definition, the two basic fields presented in Sect. 3.1 become special cases of (3): For $\Delta_{G C-M P}=1.0(\operatorname{good}$ continuation) follows $\phi(1.0)=0$ and hence (3) becomes equal to the definition of the stick field (1). For $\Delta_{G C-M P}=-1.0$ (minimal path) follows $\phi(-1.0)=\pi$ and hence (3) becomes equal to the definition of the ball field (2). Examples of the field $\mathbf{S}_{G C-M P}$ for intermediate values of the GC-MP contrast are depicted in Fig. 3 while results of their application are shown in Sect. 4.

A motivation for the definition (3) of the general stick field may also be deduced from the following considerations: Let us first consider the rotation of the inducer tokens within the (absolute) range $0 \ldots \phi\left(\Delta_{G C-M P}\right)$ from their original orientation. Such a rotation is implicitly present in the interpolation triangles of Fig. 1 where in both cases the interpolations lie inside the triangles: Therefore, the solutions for $\Delta_{G C-M P}<1$ can be achieved by rotating the inducer angles $\alpha$ and $\beta$ towards the MP-line. However, the MP-line is only available for a two-stage approach where an inducer has already been grouped with a second inducer before the actual interpolation trajectory is created. Furthermore, the use of the MP-line contradicts the concept to solely use local information at the inducer. Although polarity information is local and could be useful for Fig. 1a where the inducers are rotated towards the inside of the occluded square, a counter-example is provided by Fig. 1b. For alternate GC-MP angles one inducer has to be rotated towards the inside of the occluded shape while the other inducer has to be rotated towards the outside. Hence, for a one-stage approach which should only be based on local information it becomes necessary to rotate the inducer in both directions from the original orientation, i.e. a rotation in the range $-\phi\left(\Delta_{G C-M P}\right) \ldots \phi\left(\Delta_{G C-M P}\right)$.

\section{Results}

In [3] a set of typical inducer configurations has been proposed. First, we show results of the generalised stick field $\mathbf{S}_{G C-M P}$ on these examples. Then, we present the applica- 


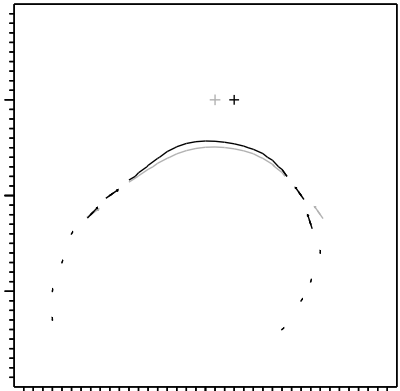

(a)

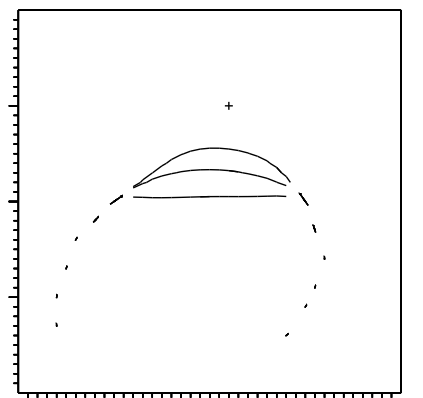

(b)

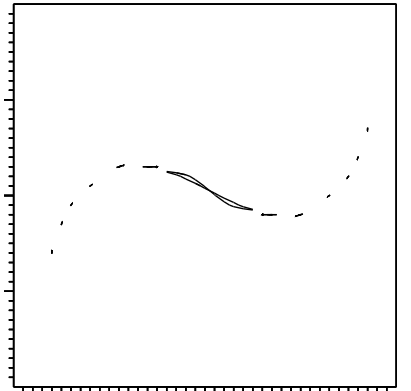

(c)

Figure 5: Curvilinear fragments: (a) Curvature information is introduced to Fig. 4a by two additional inducers. Then, the interpolated curve changes for curved inputs (black lines) in comparison to rectilinear inputs (gray lines). Crosses depict the positions of the GC-vertices, i.e. highest ball saliencies, in both cases. Dotted lines are added for visualisation only. (b) Opposite inducers: Interpolations for varying GC-MP contrasts $\Delta_{G C-M P}=-1.0,-0.6,-0.2, \ldots, 1.0$ (radii of the circles are $r_{\alpha}=15$ and $r_{\beta}=10$ ). (c) Alternate inducers: Two heterogeneous arcs with internal GC-MP angles, i.e. inside the convex region bounded by the GC-lines (for clarity only curves are depicted for $\left.\Delta_{G C-M P}=1.0,0.6\right)$.

tion of our approach to real images which have previously been used in psychophysical experiments.

In Fig. 4 the input consists of the fundamental cases created by two inducers: the opposite and the alternate configuration as in Fig. 1. While the application of the regular stick field does not change the shape of the interpolated curves, the application of the generalised stick field allows to vary the the interpolation between the GC- and the MPsolution in dependency of the newly introduced parameter $\Delta_{G C-M P}$. Note that the curves for $\Delta_{G C-M P} \rightarrow-1.0$ coincide because the increased opening angle of the stick field does not change the field significantly in the region where the grouping between the inducers occurs. Also note that, the vertex of the GC-triangle is not visible in the stick saliency for which the curves are depicted here. However, the vertex is encoded in the ball saliency component of the tensorial output (see Fig. 5a). A connection of the vertex and the curve is possible by a feature integration step as introduced in [24].

As the first example has only a single oriented token at each inducer position, no further information about curvature is given. In that case, the GC-solution will create a rectilinear interpolation due to the preference of the stick field $\mathbf{S}$ for a straight continuation. An important advantage of the tensor voting approach is the possibility to combine more than single tokens at an inducer location, which allows also to handle the completion of curvilinear fragments. Figure 5 shows how curvature may be introduced by the addition of a second token to each inducer. As depicted in this example, the shape of the interpolation changes in dependency of the presented curvature. According to the framework presented here, no changes in the processing are necessary in order to apply the interpolation to the rectilinear domain or to the curvilinear domain.

Figures 5a,b depict a configuration of opposite GC-MP angles for curvilinear inputs where the (circular) GC-lines of the two inducers intersect. Figure 5c depicts a case of curvilinear inputs in alternate configuration. (Two other alternate configurations are 


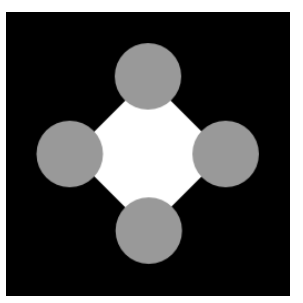

(a)

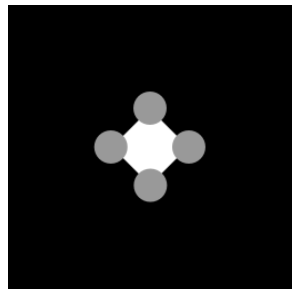

(b)

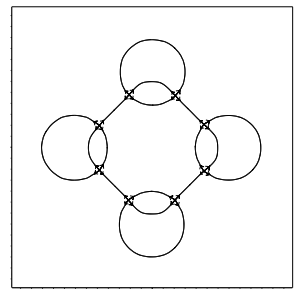

(c)

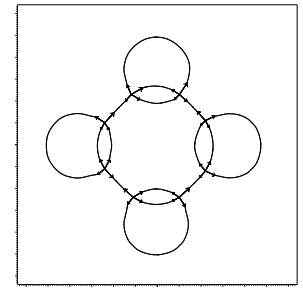

(d)

Figure 6: Influence of scale on the completion: (a), (b) Input images. (c) Resulting shapes for $\Delta_{G C-M P}=1$. (d) Resulting shapes for $\Delta_{G C-M P}=0.6$. Display enlarged by factor 2 to facilitate comparability.

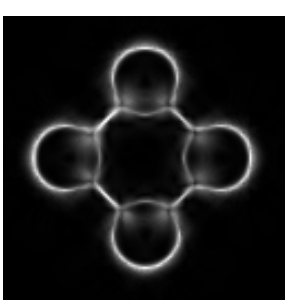

(a)

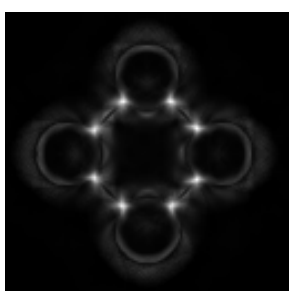

(b)

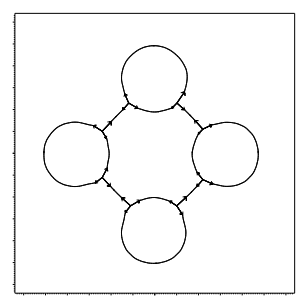

(c)

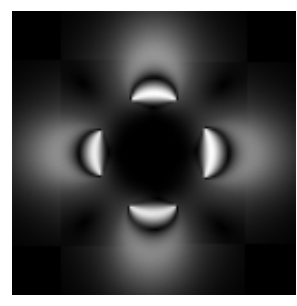

(d)

Figure 7: Intermediate results of the grouping process for Fig. $6 \mathrm{~b}$ (all images enlarged by factor 2): (a) Stick saliency map (brighter pixels correspond to higher saliencies). (b) Ball saliency map. (c) Extracted junction graph consisting of curves and junctions labelled with types (Note that the branches of the junctions are highlighted by small arrowheads). The T-junctions vote with the generalised stick field along their stems. (d) Resulting stick saliency from voting along the stems of the T-junctions $(\sigma=24)$.

possible for curvilinear inputs which yielded similar results.) All alternate configurations yield an interpolation with one point of inflection. The number of inflections is not higher because the curves leave early the GC-lines instead of sharply turning along the bow tie shape.

Figure 6 provides a more extensive example which uses images as input and shows the influence of scale on visual interpolation. For displays as in Fig. $6 a$ and b, subjects are reported to perceive the occluded square with different degrees of roundness [3]: While the larger square is completed close to the GC-solution, the smaller square yields a stronger perception of roundness, i.e. a completion closer to the MP-solution. Our approach allows to handle both cases if we measure the length of the line ending in the stems of T-junctions and compute $\Delta_{G C-M P}$ as a function of this length so that $\Delta_{G C-M P} \approx 1$ for long lines (Fig. 6c) and $\Delta_{G C-M P}<1$ for shorter lines (Fig. 6d).

This example also illustrates how grouping is accomplished by the tensor voting technique: First, the images are transfered to tensor tokens by the application of a Gabor transform. Tensor voting (with the regular fields) yields the stick saliencies and the ball saliencies depicted in Fig. 7a,b. From these maps curves and junctions are extracted and connected to the graph depicted in Fig. 7c (see [13] for details). The junctions are labelled with types according to the configurations of incoming branches. Then, the T-junctions can be used as inducers for voting with the generalised stick field with corresponding 
$\Delta_{G C-M P}$ which yields the stick saliency map shown in Fig. 7 d. From this map the completed curves are extracted and added to the graph which yields the results depicted in Fig. 6d.

\section{Discussion}

In this paper, we have extended the tensor voting framework for perceptual grouping by the integration of a GC-MP contrast parameter. The extension is achieved from a generalisation of the voting field definition. Our approach follows the important guideline to only use local information available at the inducer locations. It does not require geometric constructions, measuring of angles nor non-local influences by strongest weights emanating from the middle between the inducers as in [3]. Note that a scale measure for cases as in Fig. 6 may also be computed locally from the endpoint saliency within the tensor voting framework. In contrast to [3] and [16], our approach is a one-stage model which does not require a preceding decision for the grouping of two inducers before the actual interpolation is built. This is shown in Fig. 6 where inducers are paired as an outcome of the grouping process and not before.

In comparison to geometric compatibility criteria such as relatability [9], tensor voting determines the compatibility of curve fragments dynamically: Fragments are compatible if the combination of the voting fields yields a salient curve between the inducer locations. The solution extends the domain of the relatability constraint because interpolations are also possible for curves with inflections and for configurations with $\alpha+\beta>90^{\circ}$. However, the conditions for compatibility differ from [3] as will be detailed in a further study. Briefly, the interpolated curve does not depend on the constructibility of triangles but rather on the degree of overlay between the cones created by the stick fields of the inducers. Interpolation only takes place in these areas of overlap, whereas it is not necessary that one or both inducers fall inside the cone of the other inducer. Further work will also deal with the actual computation of the GC-MP contrast from contextual information, esp. the inclusion of endpoint saliency.

\section{References}

[1] T. Albright and G. Stoner. Contextual influences on visual processing. Annual Review of Neuroscience, 25:339-379, 2002.

[2] A. Bregman. Asking the 'what for' question in auditory perception. In M. Kubovy and J. Pomerantz, editors, Perceptual organization, pages 99-117. Erlbaum, Hillsdale, NJ, 1981.

[3] C. Fantoni and W. Gerbino. Contour interpolation by vector-field combination. $J$. Vision, 3:281-303, 2003.

[4] D. Field, A. Hayes, and R. Hess. Contour integration by the human visual system: evidence for a local "association field". Vision Res., 33:173-193, 1993.

[5] S. Fischer, P. Bayerl, H. Neumann, G. Cristóbal, and R. Redondo. Are iterations and curvature useful for tensor voting? In $\operatorname{ECCV}$ (3), pages 158-169, 2004.

[6] G. Guy and G. Medioni. Inferring global perceptual contours from local features. Int. J. Computer Vision, 20:113-133, 1996. 
[7] F. Heitger and R. v. d. Heydt. A computational model of neural contour processing: Figure-ground segregation and illusory contours. In ICCV, pages 32-40, 1993.

[8] G. Kanizsa. Organization in Vision. Praeger, New York, 1979.

[9] P. Kellman and T. Shipley. A theory of visual interpolation in object perception. Cognitive Psychology, 23:141-221, 1991.

[10] I. Kovacs. Gestalten of today: Early processing of visual contours and surfaces. Behav. Brain Res., 82:1-11, 1996.

[11] J. Larsson, K. Amunts, B. Gulyas, A. Malikovic, K. Zilles, and P. Roland. Neuronal correlates of real and illusory contour perception: functional anatomy with PET. European J. Neurosci., 11:4024-4036, 1999.

[12] T. Lee. The nature of illusory contour computation. Neuron, 33:667-668, 2002.

[13] A. Massad and G. Medioni. 2-D shape decomposition into overlapping parts. In 4th International Workshop on Visual Form (IWVF4), pages 398-409, 2001.

[14] G. Medioni, M.-S. Lee, and C.-K. Tang. A computational framework for segmentation and grouping. Elsevier, Amsterdam, 2000.

[15] J. Mendola, A. Dale, B. Fischl, A. Liu, and R. Tootell. The representation of illusory and real contours in human cortical visual areas revealed by functional magnetic resonance imaging. J. Neurosci., 19:8560-8572, 1999.

[16] P. Mordohai and G. Medioni. Junction inference and classification for figure completion using tensor voting. In IEEE Workshop Percept. Org. in Comp. Vis. (POCV), 2004.

[17] P. Parent and S. Zucker. Trace inference, curvature consistency, and curve detection. PAMI, 11:823-839, 1989.

[18] L. Pessoa and P. de Weerd, editors. Filling-In: From Perceptual Completion to Cortical Reorganization. Oxford Univ. Press, 2003.

[19] J. Pillow and N. Rubin. Perceptual completion across the vertical meridian and the role of early visual cortex. Neuron, 33:805-813, 2002.

[20] V. Ramachandran. Filling in gaps in perception: part 1. Curr. Dir. Psychol. Sci., 1:199-205, 1992.

[21] S. Sarkar and K. Boyer. Integration, inference, and management of spatial information using bayesian networks: Perceptual organization. PAMI, 15:256-274, 1993.

[22] T. Shipley and P. Kellman, editors. From Fragments to Objects - Segmentation and Grouping in Vision. Elsevier, 2001.

[23] M. Singh. Modal and amodal completion generate different shapes. Psychological Science, 15:454-459, 2004.

[24] C.-K. Tang and G. Medioni. Inferring integrated surface, curve, and junction description from sparse 3-D data sets. PAMI, 20:1206-1223, 1998.

[25] S. Ullman. Filling-in the gaps: The shape of subjective contours and a model for their generation. Biol. Cybern., 25:1-6, 1976.

[26] L. Williams and D. Jacobs. Stochastic completion fields: A neural model of illusory contour shape and salience. Neural Comp., 9:837-858, 1997. 